\title{
Article
}

\section{HER2 Status in High-Risk Endometrial Cancers (PORTEC-3): Relationship with Histotype, Molecular Classification, and Clinical Outcomes}

\author{
Lisa Vermij ${ }^{1}{ }^{1}$, Nanda Horeweg ${ }^{2}{ }^{\circledR}$, Alicia Leon-Castillo ${ }^{1}$, Tessa A. Rutten ${ }^{1}$, Linda R. Mileshkin ${ }^{3}$, \\ Helen J. Mackay ${ }^{4}$, Alexandra Leary ${ }^{5}$, Melanie E. Powell ${ }^{6}$, Naveena Singh ${ }^{7}$, Emma J. Crosbie ${ }^{8,9}$ (D, \\ Vincent T.H.B.M. Smit ${ }^{1}$, Carien L. Creutzberg ${ }^{2}$ and Tjalling Bosse ${ }^{1, *}$
}

\section{check for} updates

Citation: Vermij, L.; Horeweg, N.; Leon-Castillo, A.; Rutten, T.A.; Mileshkin, L.R.; Mackay, H.J.; Leary, A.; Powell, M.E.; Singh, N.; Crosbie, E.J.; Smit, V.T.; et al. HER2 Status in High-Risk Endometrial Cancers (PORTEC-3): Relationship with Histotype, Molecular Classification, and Clinical Outcomes. Cancers 2021, 13, 44. https://dx.doi.org/10.3390/ cancers13010044

Received: 9 December 2020 Accepted: 22 December 2020 Published: 25 December 2020

Publisher's Note: MDPI stays neutral with regard to jurisdictional claims in published maps and institutional affiliations.

Copyright: () 2020 by the authors. Licensee MDPI, Basel, Switzerland. This article is an open access article distributed under the terms and conditions of the Creative Commons Attribution (CC BY) license (https: / / creativecommons.org/ licenses/by/4.0/).
1 Department of Pathology, Leiden University Medical Center, 2300 RC Leiden, The Netherlands; L.Vermij@lumc.nl (L.V.); A.Leon_del_Castillo@lumc.nl (A.L.C.); T.A.Rutten@lumc.nl (T.A.R.); V.T.H.B.M.Smit@lumc.nl (V.T.H.B.M.S.)

2 Department of Radiation Oncology, Leiden University Medical Center, 2300 RC Leiden, The Netherlands; N.Horeweg@lumc.nl (N.H.); C.L.Creutzberg@lumc.nl (C.L.C.)

3 Division of Cancer Medicine, Peter MacCallum Cancer Centre, Melbourne, VIC 3000, Australia; Linda.Mileshkin@petermac.org

4 Division of Medical Oncology and Hematology, Sunnybrook Odette Cancer Centre, Toronto, ON M4N 3M5, Canada; helen.mackay@sunnybrook.ca

5 Department of Medical Oncology, Gustave Roussy, 94805 Villejuif, France; Alexandra.LEARY@gustaveroussy.fr

6 Department of Clinical Oncology, Barts Health NHS Trust, London E1 1BB, UK; melanie.powell10@nhs.net

7 Department of Pathology, Barts Health NHS Trust, London E1 1BB, UK; N.Singh@bartsandthelondon.nhs.uk

8 Division of Cancer Sciences, University of Manchester, St Mary's Hospital, Manchester M13 9WL, UK; Emma.Crosbie@manchester.ac.uk

9 Department of Obstetrics and Gynecology, Manchester University NHS Foundation Trust, Manchester Academic Health Science Centre, Manchester M13 9NQ, UK

* Correspondence: T.Bosse@lumc.nl; Tel.: +31-071-526-6639

Simple Summary: HER2 testing in endometrial cancer (EC) has gained renewed interest as a therapeutic target. However, HER2 status has not been investigated in the context of the molecular EC classification. Here, we aimed to determine the clinicopathological features and prognostic significance of the HER2 status in the molecularly classified PORTEC-3 trial population of patients with high-risk EC. HER2 status of 407 high-risk EC was determined by HER2 immunohistochemistry and HER2 dual in situ hybridization. Twenty-four (5.9\%) HER2-positive EC of various histological subtypes were identified, including serous $(n=9,37.5 \%)$, endometrioid $(n=6,25.0 \%)$, and clear cell $(n=5,20.8 \%$ ). HER2 positivity was highly associated with the p53-abnormal subgroup (p53abn, $23 / 24$ cases; $p<0.0001)$. The correlation between $p 53 a b n$ and the HER2 status $(\rho=0.438 ; p<0.0001)$ was significantly stronger $(p<0.0001)$ than between serous histology and the HER2 status $(\rho=0.154$; $p=0.002$ ). HER2 status did not have independent prognostic value for survival after correction for the molecular classification. Our study strongly suggests that molecular subclass-directed HER2 testing is superior to histotype-directed testing.

Abstract: HER2 status has not been investigated in the context of the molecular endometrial cancer (EC) classification. Here, we aimed to determine the clinicopathological features and prognostic significance of the HER2 status in the molecularly classified PORTEC-3 trial population of patients with high-risk EC (HREC). HER2 testing was performed on tumor tissues of 407 molecularly classified HREC. HER2 status was determined by HER2 immunohistochemistry (IHC; all cases) and subsequent HER2 dual in situ hybridization for cases with any (in) complete moderate to strong membranous HER2 IHC expression. The $X^{2}$ test and Spearman's Rho correlation coefficient were used to compare clinicopathological and molecular features. The Kaplan-Meier method, log-rank test, and Cox proportional hazards models were used for survival analysis. We identified 24 (5.9\%) HER2-positive EC of various histological subtypes including serous $(n=9,37.5 \%)$, endometrioid $(n=6,25.0 \%)$, and clear cell $(n=5,20.8 \%)$. HER2 positivity was highly associated with the p53-abnormal subgroup 
(p53abn, 23/24 cases; $p<0.0001)$. The correlation between p53abn and the HER2 status $(\rho=0.438$; $p<0.0001)$ was significantly stronger $(p<0.0001)$ than between serous histology and the HER2 status $(\rho=0.154 ; p=0.002)$. HER2 status did not have independent prognostic value for survival after correction for the molecular classification. Our study strongly suggests that molecular subclassdirected HER2 testing is superior to histotype-directed testing. This insight will be relevant for future trials targeting HER2.

Keywords: endometrial cancer; high-risk; HER2; ERBB2; p53

\section{Introduction}

Endometrial cancer (EC) is the most common gynecological cancer in developed countries and is primarily treated with surgery. Around $15-20 \%$ of women with EC have high-risk disease with increased incidence of distant metastases and cancer-related death High-risk features include advanced age, high-grade non-endometrioid histology, substantial lymphovascular space invasion (LVSI), and advanced stage disease. For many years, standard adjuvant treatment for women with high-risk EC has been pelvic external beam radiotherapy (EBRT) [1]. The international randomized Adjuvant Chemoradiotherapy Versus Radiotherapy Alone in Women with High-Risk Endometrial Cancer (PORTEC-3) trial investigated the benefit of adjuvant chemotherapy in combination with EBRT (chemoradiotherapy (CTRT)) versus EBRT alone (radiotherapy (RT)) in patients with high-risk EC [2]. A small but significant benefit was shown in overall survival (OS) and failure-free survival (FFS) in favor of the CTRT treatment arm, albeit at the cost of significantly toxicity. Thus, the identification of patients who will benefit from chemotherapy is essential. However, the assessment of high-risk pathologic features is subject to a substantial interobserver disagreement, complicating the identification of this subset of patients [3,4].

Over the last decade, the Cancer Genome Atlas (TCGA)-based molecular EC classification has proven to have significant prognostic value in EC patients with the potential to refine current clinicopathological risk assessment [5-8]. The molecular classification stratifies EC into four distinct molecular subgroups including: (1) POLE-ultramutated (POLEmut) EC which are characterized by pathogenic mutations in the exonuclease domain of POLE, associated with endometrioid histology, and have an excellent prognosis, (2) mismatch repair-deficient (MMRd) EC which are characterized by microsatellite instability, are exclusive to endometrioid histology, and show an intermediate prognosis, (3) p53-abnormal (p53abn) EC which are associated with somatic copy number alterations (SCNA), high-grade endometrioid and serous histology, and have a poor prognosis, (4) nospecific-molecular-profile (NSMP) EC which are microsatellite-stable and have low SCNA, associated with low-grade endometrioid histology and have an intermediate prognosis. Furthermore, the molecular classification provides a strong biological basis for future subclass-specific clinical trials. Defining therapeutic targets within the p53abn EC subgroup should be prioritized given its poor prognosis despite adjuvant CTRT [9].

Human epidermal growth factor receptor 2 (HER2), encoded by the ERBB2 gene, has been established as an important biomarker with both prognostic and therapeutic implications in breast and gastric cancers [10-12]. HER2-positive breast cancers are associated with aggressive disease course, poor prognosis, and show a strong correlation with other independent prognostic markers such as histologic subtype and grade [13]. Trastuzumab, a monoclonal antibody directed against HER2, has proven to increase survival of patients with HER2-positive breast cancer and of those with advanced and metastatic gastric cancer. This has subsequently led to the approval of trastuzumab therapy in these cancers by the Food and Drug Association (FDA) [14-17].

HER2 protein overexpression and/or ERBB2 amplifications have also been described in EC, albeit with conflicting estimates of frequencies and clinical outcomes. Possibly, this is due to a lack of universal HER2 testing and scoring methods and differences in 
histological subtypes included in the studies [18-21]. Nevertheless, existing evidence points towards a correlation between HER2 protein overexpression and/or ERBB2 amplification in EC with serous histology (SEC), with reported frequencies ranging between 29-49\% [18-21]. Recently, a phase II clinical trial comparing adjuvant carboplatinpaclitaxel with and without trastuzumab in 58 patients with advanced and recurrent HER2-overexpressing SEC showed increased progression-free survival in favor of the combined chemotherapy-trastuzumab treatment arm [22,23]. These findings encourage further investigation of the efficacy of trastuzumab in combination with chemotherapeutic agents in HER2-overexpressing/amplified EC.

Although the clinical and therapeutic implications of HER2 overexpression/amplification in EC have been studied over the last decade, it has not yet been investigated in the context of the molecular EC classification. We hypothesize that molecular subgroup-directed screening for HER2 status is more effective in identifying patients who may benefit from anti-HER2 therapies than histology-directed screening for HER2 status. In addition, the presence of PIK3CA mutations and/or PTEN loss have been associated with trastuzumab resistance in HER2-positive breast cancers, [24-26]. Previous studies investigating the efficacy of trastuzumab in HER2-overexpressing EC did not take this into account, possibly (negatively) influencing clinical trial outcomes. Gaining more insight in the prevalence of PIK3CA and PTEN mutations in HER2-positive EC can provide essential information about the magnitude of this trastuzumab resistance mechanism in EC.

The molecular EC classification is currently being implemented in clinical practice [27]. Now that anti-HER2 treatments in EC patients show promising results, it is time to study HER2 more comprehensively. Therefore, we aimed to investigate the association between the HER2 amplification status and clinicopathological and molecular features as well as patient outcomes in the well-defined PORTEC-3 trial cohort of high-risk EC supplemented by publicly available TCGA data. To our knowledge, this is the first comprehensive study investigating the correlation between the HER2 amplification status and the molecular EC classification.

\section{Results}

\subsection{Clinicopathological Characteristics}

HER2 status could be determined for 405 (98.8\%) molecularly classified ECs from PORTEC-3 (Figure 1). For three cases, there was insufficient material available for HER2 testing. In two cases, HER2 IHC was successful, but the final HER2 status could not be determined due to failed HER2 dual in situ hybridization (DISH) testing and inadequate quality of the copy number plot generated by next-generation sequencing (NGS). The median follow-up at the time of analysis was 6.1 years (95\% CI: $5.86-6.27 \%$ ).

Of all the 405 evaluable cases, 263 EC (64.6\%) did not have any membranous HER2 immunoreactivity and 45 EC (11.1\%) showed faint membranous HER2 IHC expression. Moderate and strong membranous immunoreactivity was observed in 60 EC $(14.7 \%)$ and 26 EC $(6.4 \%)$, respectively. In 13 cases (3.2\%), HER2 IHC could not be reliably interpreted due to the absence of a positive external control. After performing DISH on the latter three groups, we identified 24 HER2-positive EC (5.9\%; 95\% CI: 3.6-8.2\%) (Figure 1). Subclonal HER2 expression was observed in 81 cases (20\%), including 11 HER2-positive EC (45.8\%). Incomplete membranous immunoreactivity was seen in 101 tumors $(24.9 \%)$, including 6 HER2-positive EC (25.0\%). All HER2 IHC slides were additionally scored by an adjusted version of the American Society of Clinical Oncology and College of American Pathologists (ASCO/CAP) 2007 guideline for breast cancer. The HER2 IHC results were compared to the HER2 amplification status by DISH (data shown in Table S2).

Age, histology, and tumor grade differed significantly between patients with HER2positive and HER2-negative EC (Table 1). Interestingly, the majority of the HER2-positive EC were of non-serous histology, including endometrioid EC $(n=6 ; 25.0 \%)$ and clear cell EC $(n=5 ; 20.8 \%)$ (Figure 2). There were no significant differences between HER2-positive 
and HER2-negative cases in stage, presence of LVSI, and adjuvant treatment received (RT vs. CTRT).

\subsection{Association with Molecular Classification and Other Molecular Alterations}

The prevalence of HER2-positive EC both in the PORTEC-3 as well as in the TCGA cohort was not evenly distributed between the four molecular subgroups $(p<0.0001)$ (Table 2). In PORTEC-3, all but one (95.8\%) of the HER2-positive ECs were classified as p53abn. Given this strong association, we additionally performed DISH on all p53abn EC with absent or any faint membranous staining to ascertain the HER2 status assignment by IHC was correct. In correspondence to the IHC results, none of these p53abn EC showed evidence of HER2 amplification. The only HER2-positive case in PORTEC-3 which was not p53abn was an NSMP EC. Examination of this case revealed a convincing wildtype p53-IHC pattern and no TP53 mutation was found by NGS analysis. The tumor showed moderate incomplete membranous HER2 immunoreactivity in $>10 \%$ of the tumor and low-level HER2 amplification by DISH (HER2:CEP17 ratio: 2.2, average HER2 count: 3.7). All POLEmut EC $(n=52)$ and MMRd EC $(n=135)$ were HER2-negative. Within the TCGA cohort, all ERBB2-amplified EC were copy number-high (CN-high). NGS analysis was successful in 18 out of the 24 HER2-positive cases in PORTEC-3. In these cases, pathogenic PIK3CA mutations were present in five (27.8\%) cases and only one HER2-positive tumor (5.6\%) showed a pathogenic PTEN frameshift mutation (c.309del; p.C105fs). In the TCGA cohort, ten out of the 25 HER2-positive cases (40.0\%) had a pathogenic PIK3CA mutation, while none of the cases had a PTEN mutation. We found a moderately strong positive correlation between HER2-positivity and the p53abn subgroup $(\rho=0.441, p<0.0001)$, which explains $19.5 \%$ of the shared variance. This correlation was significantly stronger $(p<0.0001)$ than the weak positive correlation that was found between HER2 positivity and serous histology $(\rho=0.154, p=0.002)$ that only explains $2.4 \%$ of the shared variance.

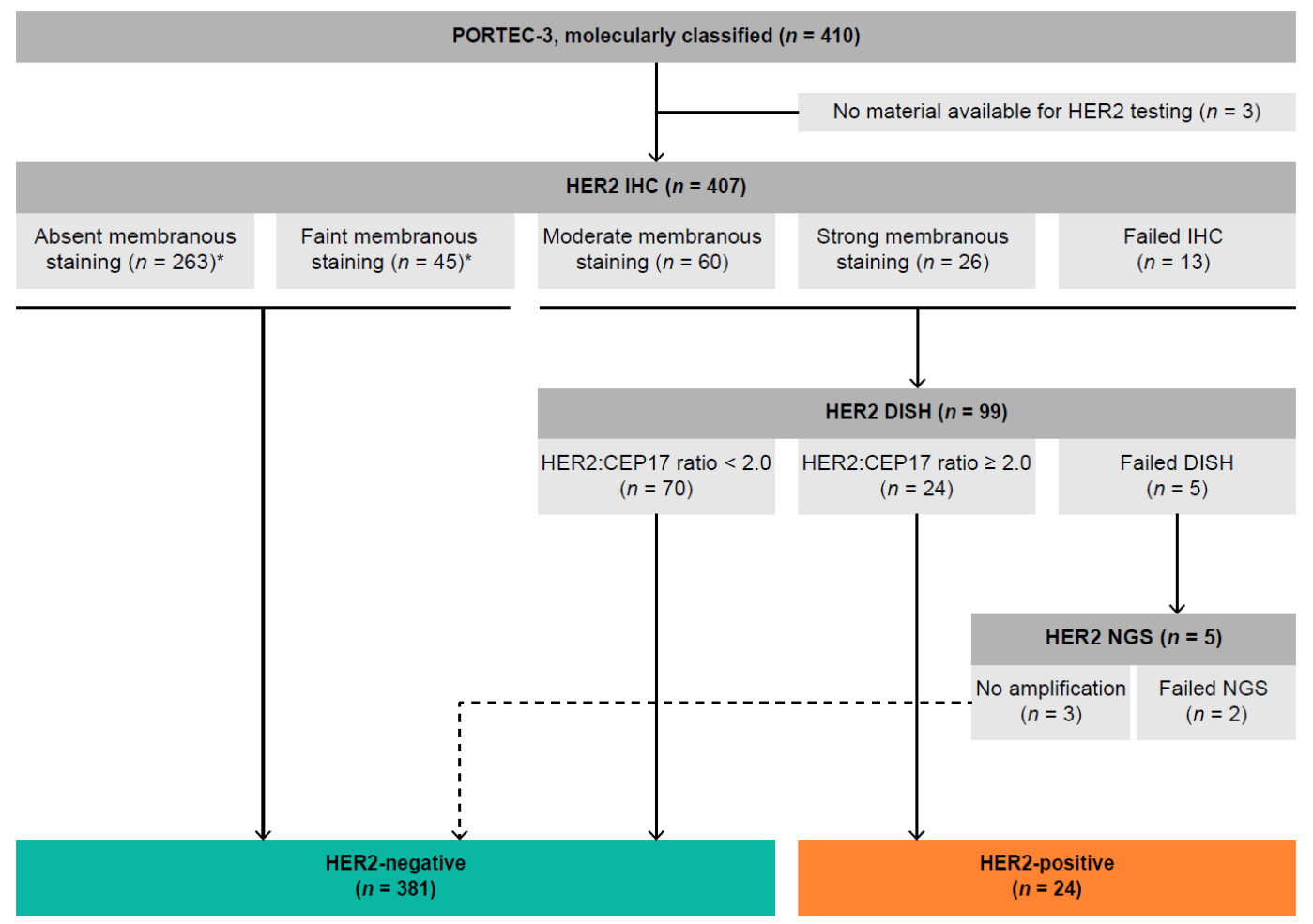

Figure 1. Flowchart of HER2 testing of molecularly classified PORTEC-3 trial participants. * SISH was performed on a random selection of cases with absent $(n=10)$ and faint $(n=10)$ membranous staining to confirm the absence of HER2 amplification. Abbreviations: IHC, immunohistochemistry; SISH, silver in situ hybridization; NGS, next-generation sequencing. 
Table 1. Patient, tumor, and treatment characteristics by HER2 status.

\begin{tabular}{|c|c|c|c|c|}
\hline Characteristic & Total & HER2-Negative & HER2-Positive & $p$-Value \\
\hline & $n=405(100 \%)$ & $n=381(94.1 \%)$ & $n=24(5.9 \%)$ & \\
\hline Age, years & & & & $<0.0001$ \\
\hline Mean (range) & $61.2(26.7-80.5)$ & $60.8(26.7-78.6)$ & $68.3(55.8-80.5)$ & \\
\hline Histotype & & & & $<0.0001$ \\
\hline Endometrioid & $272(67.2)$ & $266(69.8)$ & $6(25.0)$ & \\
\hline Serous & $64(15.8)$ & $55(14.4)$ & $9(37.5)$ & \\
\hline Clear cell & $39(9.6)$ & $34(8.9)$ & $5(20.8)$ & \\
\hline Mixed (EEC-S) & $9(2.2)$ & $8(2.1)$ & $1(4.2)$ & \\
\hline Mixed (EEC-CCC) & $9(2.2)$ & $8(2.1)$ & $1(4.2)$ & \\
\hline Other & $12(3.0)$ & $10(2.6)$ & $2(8.3)$ & \\
\hline Grade & & & & $<0.0001$ \\
\hline $1-2$ & $163(40.2)$ & $162(42.5)$ & $1(4.2)$ & \\
\hline 3 & $242(59.8)$ & $219(57.5)$ & $23(95.8)$ & \\
\hline Stage & & & & 0.080 \\
\hline IA & $53(13.1)$ & $47(12.3)$ & $6(25.0)$ & \\
\hline IB & $73(18.0)$ & $68(17.8)$ & $5(20.8)$ & \\
\hline II & $102(25.2)$ & $96(25.2)$ & $6(25.0)$ & \\
\hline IIIA & $45(11.1)$ & $44(11.5)$ & $1(4.2)$ & \\
\hline IIIB & $29(7.2)$ & $27(7.1)$ & $2(8.3)$ & \\
\hline IIIC & $103(25.4)$ & $99(26.0)$ & $4(16.7)$ & \\
\hline LVSI & & & & 0.38 \\
\hline Absent & $152(37.5)$ & $145(38.1)$ & $7(29.2)$ & \\
\hline Present & $253(62.5)$ & $236(61.9)$ & $17(70.8)$ & \\
\hline Lymphadenectomy & & & & 0.64 \\
\hline No & $184(45.4)$ & $172(45.1)$ & $12(50.0)$ & \\
\hline Yes & $221(54.6)$ & 209 (54.9) & $12(50.0)$ & \\
\hline Treatment received & & & & 0.45 \\
\hline RT & $199(49.1)$ & $189(49.6)$ & $10(41.7)$ & \\
\hline CTRT & $206(50.9)$ & $192(50.4)$ & $14(58.3)$ & \\
\hline
\end{tabular}

Abbreviations: EEC-S, mixed endometrioid and serous endometrial cancer; EEC-CCC, mixed endometrioid and clear cell endometrial cancer; RT, radiotherapy; CTRT, chemoradiotherapy.

\subsection{Association between HER2 Status and Clinical Outcome}

We analyzed differences in recurrence-free survival (RFS) and OS for women with and without HER2-positive EC in the PORTEC-3 cohort. Patients with HER2-positive EC had significantly poorer survival than patients with HER2-negative EC (5-year RFS: $42.9 \%$ vs. $73.6 \%, p<0.0001 ; 5$-year OS: $42.3 \%$ vs. $81.4 \%, p<0.0001$ ) (Figure 3A,B). However, in a subanalysis including only p53abn EC, there was no difference in prognosis (Figure 3C,D). The prognostic value of the HER2 status was evaluated in univariable and multivariable analysis including all PORTEC-3 patients (Table 3). In univariable analysis, HER2 positivity was associated with significantly worse recurrence-free survival (HR: 2.76; 95\% CI:1.55-4.93) and overall survival (HR: 3.64; 95\% CI: 2.02-6.58). However, when corrected for age, stage, grade, LVSI, and the molecular classification by multivariable analysis, HER2 status was not an independent prognostic factor for both RFS (HR: 1.15; 95\% CI: 0.60-2.12) and OS (HR: 1.24; 95\% CI: 0.63-2.42).

These analyses were also performed for the combined PORTEC-3 and TCGA cohort confirming the results reported above (Table S1, Figure S1). 

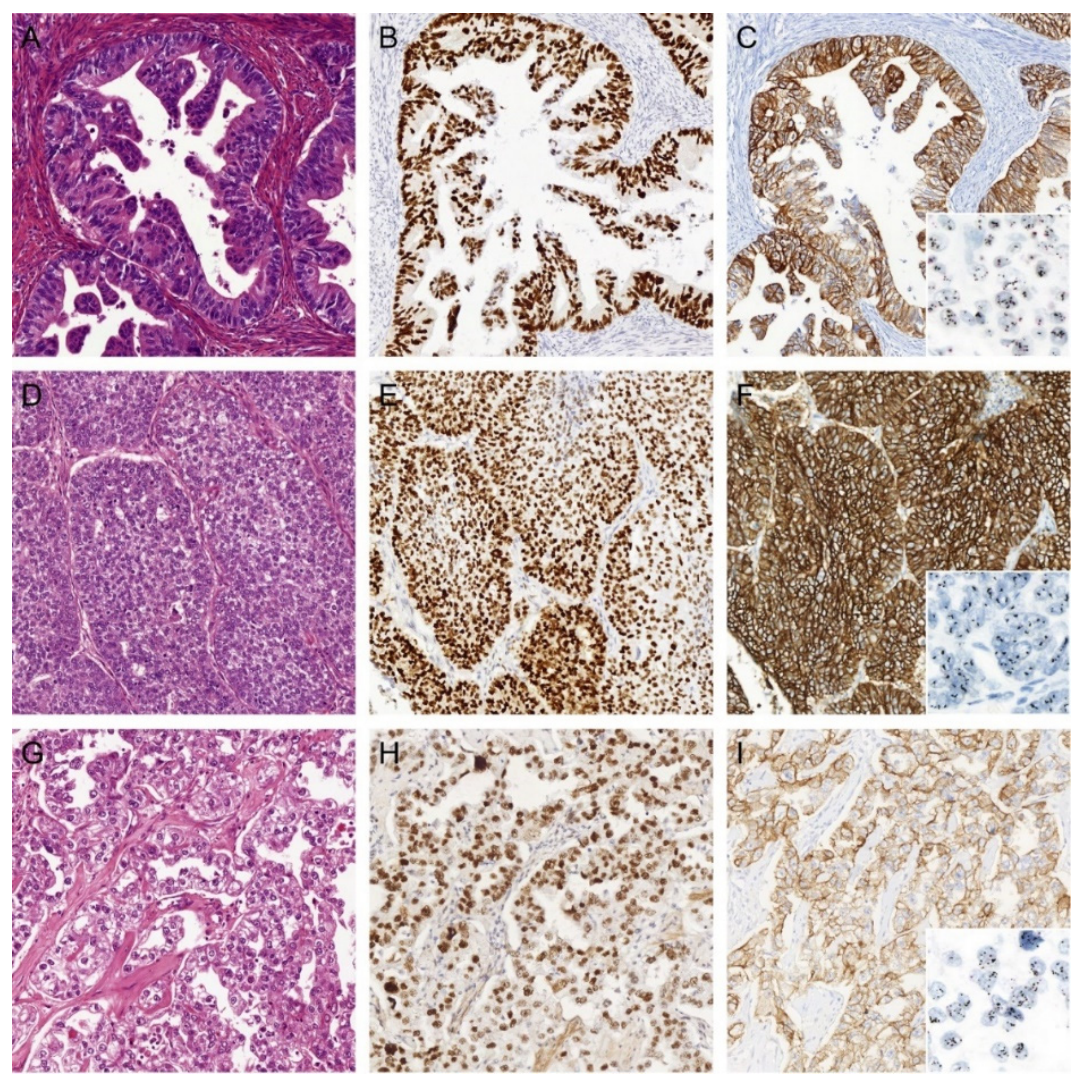

Figure 2. Three examples of HER2-positive endometrial cancers (EC) of non-serous histology. (A) H\&E of an EC diagnosed as International Federation of Gynecology and Obstetrics (FIGO) grade 2 endometrioid EC (EEC) with (B) aberrant mutant-like p53 immunostaining, (C) strong (in)complete membranous HER2 immunostaining, and HER2 gene amplification by dual in situ hybridization (DISH). (D) H\&E of an EC diagnosed as FIGO grade 3 EEC with (E) aberrant mutant-like p53 immunostaining, (F) strong complete membranous HER2 immunostaining, and HER2 gene amplification by DISH. (G) H\&E of an EC diagnosed as endometrial clear cell carcinoma with (H) aberrant mutant-like p53 immunostaining, (I) strong complete membranous HER2 immunostaining, and HER2 gene amplification by DISH. All images $\times 20$ objective magnification.

Table 2. Association between the HER2 status and molecular EC classification.

\begin{tabular}{|c|c|c|c|c|c|c|}
\hline \multirow[t]{2}{*}{ Cohort } & \multirow[b]{2}{*}{$\begin{array}{c}\text { Total } \\
n=405\end{array}$} & \multicolumn{5}{|c|}{ Molecular Subgroup } \\
\hline & & $\begin{array}{c}\text { POLEmut } \\
n=52(12.8 \%)\end{array}$ & $\begin{array}{c}\text { MMRd } \\
n=135(33.3 \%)\end{array}$ & $\begin{array}{c}\text { NSMP } \\
n=126(31.1 \%)\end{array}$ & $\begin{array}{c}\text { P53 } \\
n=92(22.7 \%)\end{array}$ & $p$-Value \\
\hline PORTEC-3 & & & & & & $<0.0001$ \\
\hline HER2-negative & $381(94.1)$ & $52(100.0)$ & $135(100.0)$ & $125(99.2)$ & $69(75.0)$ & \\
\hline \multirow[t]{2}{*}{ HER2-positive } & $24(5.9)$ & $0(0.0)$ & $0(0.0)$ & $1(0.8)$ & $23(25.0)$ & \\
\hline & $\begin{array}{c}\text { Total } \\
n=506\end{array}$ & $\begin{array}{c}\text { POLEmut } \\
n=49(9.7 \%)\end{array}$ & $\begin{array}{c}\text { MSI } \\
n=148(29.2 \%)\end{array}$ & $\begin{array}{c}\text { CN-low } \\
n=146(28.9 \%)\end{array}$ & $\begin{array}{c}\text { CN-high } \\
n=163(32.3 \%)\end{array}$ & $p$-Value \\
\hline UCEC TCGA PanCancer & & & & & & $<0.0001$ \\
\hline Non-ERBB2-amplified & $481(95.1)$ & $49(100.0)$ & $148(100.0)$ & $146(100.0)$ & $138(84.7)$ & \\
\hline ERBB2-amplified & $25(4.9)$ & $0(0.0)$ & $0(0.0)$ & $0(0.0)$ & $25(15.3)$ & \\
\hline
\end{tabular}

Abbreviations: POLEmut, POLE-(ultra-)mutated; MMRd, mismatch repair-deficient; NSMP, no specific molecular profile; p53abn, p53-abnormal; TCGA, the Cancer Genome Atlas; UCEC, uterine corpus endometrial carcinoma; MSI, microsatellite-unstable; CN-low, copy number-low; CN-high, copy number-high. 
A

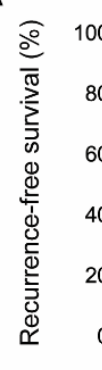

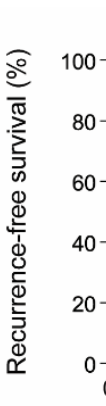

No. at risk
HER2-negative 38
HER2-positive 24

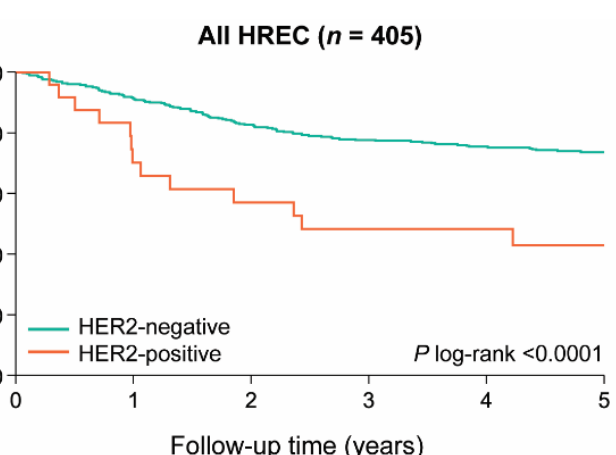

Follow-up time (years)

C

के

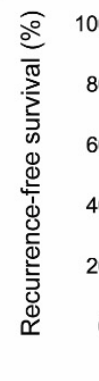

No. at risk

HER2-negative 69

$$
\begin{array}{cccc}
347 & 314 & 289 & 267 \\
16 & 13 & 11 & 10
\end{array}
$$

p53abn HREC $(n=92)$

Figure 3. Kaplan-Meier survival curves for 5-year (A) recurrence-free survival (RFS) and (B) overall survival (OS) for PORTEC-3 patients with HER2-positive and HER2-negative high-risk endometrial cancer (HREC); 5-year (C) RFS and (D) OS for patients with HER2-positive and HER2-negative p53 abnormal HREC.

Table 3. Multivariable analysis of the HER2 status and clinicopathological features in high-risk endometrial cancers $(n=$

\begin{tabular}{|c|c|c|c|c|c|c|c|}
\hline \multirow[t]{3}{*}{ Characteristic } & \multirow[b]{3}{*}{ Total $\mathbf{n}$} & \multicolumn{3}{|c|}{ Recurrence-Free Survival } & \multicolumn{3}{|c|}{ Overall Survival } \\
\hline & & \multicolumn{3}{|c|}{118 Events } & \multicolumn{3}{|c|}{92 Events } \\
\hline & & HR & $95 \% \mathrm{CI}$ & p-Value & HR & $95 \% \mathrm{CI}$ & $p$-Value \\
\hline Age & 405 & 1.028 & $1.003-1.054$ & 0.030 & 1.056 & $1.025-1.088$ & $<0.0001$ \\
\hline \multicolumn{8}{|l|}{ HER2 status } \\
\hline Negative & 381 & 1 & & & 1 & & \\
\hline Positive & 24 & 1.150 & $0.596-2.220$ & 0.68 & 1.237 & $0.632-2.419$ & 0.54 \\
\hline \multicolumn{8}{|l|}{ Molecular subgroups } \\
\hline MMRd & 135 & 1 & & & 1 & & \\
\hline p53abn & 92 & 2.720 & $1.594-4.639$ & 0.000 & 2.297 & $1.296-4.071$ & 0.004 \\
\hline POLEmut & 52 & 0.085 & $0.011-0.625$ & 0.015 & 0.106 & $0.014-0.789$ & 0.028 \\
\hline NSMP & 126 & 0.984 & $0.601-1.612$ & 0.95 & 0.600 & $0.323-1.112$ & 0.11 \\
\hline \multicolumn{8}{|l|}{ Histology and grade } \\
\hline Endometrioid, low grade & 160 & 1 & & & 1 & & \\
\hline Endometrioid, high grade & 112 & 1.086 & $0.637-1.852$ & 0.76 & 1.353 & $0.734-2.494$ & 0.33 \\
\hline Non-endometrioid & 133 & 0.842 & $0.476-1.490$ & 0.55 & 1.015 & $0.532-1.936$ & 0.97 \\
\hline \multicolumn{8}{|l|}{ Stage } \\
\hline I-II & 228 & 1 & & & 1 & & \\
\hline III & 177 & 2.047 & $1.374-3.048$ & 0.030 & 1.826 & $1.174-2.841$ & 0.008 \\
\hline \multicolumn{8}{|l|}{ LVSI } \\
\hline Absent & 152 & 1 & & & 1 & & \\
\hline Present & 253 & 1.281 & $0.838-1.957$ & 0.25 & 1.173 & $0.720-1.909$ & 0.52 \\
\hline
\end{tabular}
405).

Abbreviations: HR, hazard ratio; POLEmut, POLE-(ultra-)mutated; MMRd, mismatch repair-deficient; NSMP, no specific molecular profile; p53abn, p53-abnormal; LVSI, lymphovascular space invasion.
B

D

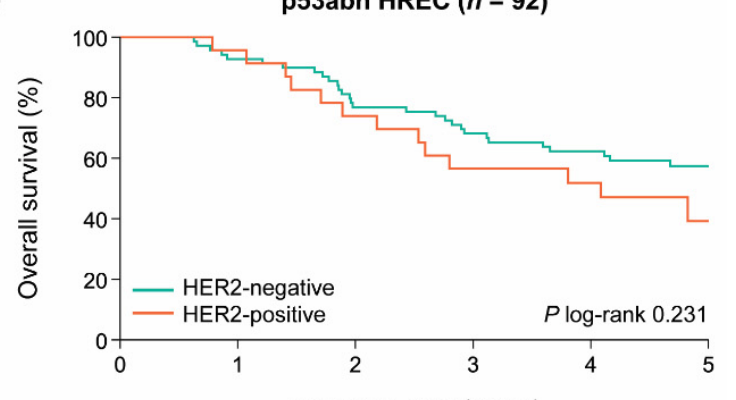

Follow-up time (years)

No. at risk

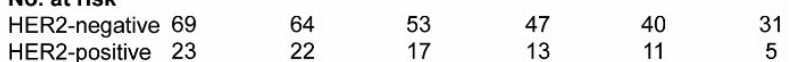




\section{Discussion}

In this study, we demonstrate that HER2 positivity is prevalent in $5.9 \%$ (95\% CI: $3.6-8.2 \%$ ) of HREC and arises in various histological subtypes including endometrioid $(25.0 \%)$, serous $(37.5 \%)$, and clear cell $(20.8 \%)$ EC. To our knowledge, this is the first study to investigate HER2 status in the context of the molecular EC classification.

HER2 positivity is strongly associated with the p53abn $(p<0.0001)$ molecular subgroup; $95.8 \%$ of all the HER2-positive ECs were identified in this molecular subgroup. This strong association was confirmed in the TCGA cohort; all ERBB2-amplified ECs were $\mathrm{CN}$-high $(p<0.0001)$. The association between HER2 positivity and the p53abn subgroup is also reflected in the survival analyses. Significantly lower 5-year RFS and OS were found in patients with HER2-positive versus HER2-negative EC (42.9\% vs. 73.6\%, $p<0.0001$ for RFS; $42.3 \%$ vs. $81.4 \%, p<0.0001$ for OS). These differences lost their statistical significance when corrected for p53 status ( $40.2 \%$ vs. $51.3 \%, p=0.313$ for RFS; $39.3 \%$ vs. $57.4 \%, p=0.231$ for OS). In addition, HER2 status did not have independent prognostic value in multivariable analysis with the established prognostic factors, including the molecular EC classification.

We are the first to report on a highly significant association between HER2 overexpression and/or amplification and the p53abn molecular subgroup $(p<0.0001)$ with $95.8 \%$ of HER2-positive EC classified in the p53abn subgroup in the PORTEC-3 HREC. This was confirmed by the similarly strong association between ERBB2 amplification and the $\mathrm{CN}$-high molecular subgroup $(p<0.0001)$ in the TCGA cohort, with $100 \%$ of the ERBB2-amplified EC classified in the CN-high subgroup. Importantly, we found that the correlation between HER2 positivity and p53abn EC is significantly stronger than the correlation between HER2 positivity and serous histology $(p<0.0001)$. The interaction between HER2 overexpression and mutant p53 status has been described in other cancers. A study using cancer cell lines with introduced gain-of-function TP53 mutations found an increase in HER2 mRNA expression levels as well as HER2 protein levels [28]. Analysis of the publicly available breast cancer dataset (GSE22358) confirm significantly higher HER2 mRNA levels in p53 mutant samples $(p=0.046)$ compared to p53 wildtype samples [29]. Mechanistically, mutant p53 has shown to be associated with enhanced transcriptional activity of HSF1 (heat shock transcription factor 1) that targets chaperon Hsp90 which in turn stabilizes the HER2 and 553 proteins, thereby further reinforcing oncogenic signaling [30]. Another study has shown an increased risk of recurrence in breast cancer patients harboring both a somatic TP53 mutation and ERBB2 amplification compared to patients with either one or no alterations [31]. Our study is one of the largest studies investigating HER2 amplification status in a cohort of HREC which were not selected on the serous histological subtype. In 2006, Morrison et al. investigated HER2 protein overexpression by IHC and ERBB2 amplification by FISH in a cohort of 483 ECs [20]. HER2 protein overexpression was reported in $14.2 \%$ $(69 / 483)$ and ERBB2 amplification was found in 6.6\% (32/483) of cases, respectively. Comparable to our findings, $46.9 \%$ of all HER2-positive EC tested by FISH were of non-serous histology (9/32: endometrioid, 17/32: serous, 2/32: clear cell carcinomas, and 3/32: other histotypes). In our study, HER2 overexpression and/or amplification was found in $14.1 \%$ of serous EC. Other studies including only serous EC reported HER2 overexpression by IHC ranging between $14 \%$ and $80 \%$ and ERBB2 amplification by FISH ranging from $21 \%$ to $47 \%$ [32].

Previously reported frequencies vary widely, possibly due to differences in the composition of study cohorts and differences in the HER2 testing methods used in the absence of an EC-specific HER2 testing method. In our cohort, HER2 amplification status was confirmed by performing DISH on all tumors with any moderate/strong HER2 IHC expression rather than applying a HER2 testing algorithm validated on breast of gastric cancers. Incomplete membranous HER2 expression was observed in 25\% $(n=6)$ of HER2-amplified EC. According to the latest ASCO/CAP 2018 HER2 Testing in Breast Cancer Guideline, these cases would have been interpreted as HER2-negative (IHC 1+) without additional in situ hybridization (ISH) testing [33]. This suggests that the current ASCO/CAP guideline for breast cancer may not be directly applicable for the identification of HER2-positive EC. 
Furthermore, in contrast to breast and gastric HER2 testing guidelines, we did not apply a threshold for the percentage of tumor cells (e.g., $>10 \%,>30 \%$ ) with moderate/strong HER2 IHC expression required to perform DISH, as such threshold is still arbitrary in EC. It is conceivable that small HER2-positive subclones may still have relevant impact on the biological behavior of a tumor. We foresee that a clinically meaningful threshold for HER2 status determination in EC will evolve from trials using anti-HER2 therapies. Given the small number of cases with $<10 \%$ HER2 positivity in our study $(n=2 / 24,8.3 \%)$, a threshold of $10 \%$ may be a good starting point going forward.

In our study, we did not detect a statistically significant difference in survival between patients with HER2-positive and HER2-negative EC after adjustment for p53 status. However, our study was not powered to detect small differences in prognosis. We can therefore not rule out that HER2 status has minor negative prognostic impact in HREC. Importantly, after extending the PORTEC-3 dataset with the TCGA cohort, we still did not find a significant independent prognostic value of the HER2 status in multivariable analysis (including the molecular classification). This makes it less likely that there is a small prognostic difference that we did not detect. Although previous studies showed prognostic value of HER2 overexpression and/or amplification in EC [19], these findings may have been confounded by an underlying mutant p53 status.

Earlier clinical studies incorporating trastuzumab as a single agent for the treatment of HER2-overexpressing and/or ERRB2-amplified EC did not show any prognostic benefit $[34,35]$. However, a phase II randomized clinical trial comparing carboplatinpaclitaxel with and without trastuzumab in 58 patients with primary stage III/IV or recurrent serous EC showed a significantly prolonged PFS with four months in patients who received combined chemotherapy-trastuzumab treatment [22,23]. These findings encourage further exploration of combined chemotherapy with anti-HER2 therapeutics in HER2-positive EC. Of interest, recently published survival analysis of p53abn EC (of all histotypes) in PORTEC-3 showed a significant benefit in 5-year RFS when chemotherapy was added to adjuvant radiotherapy (5-year RFS of $58.6 \%$ with CTRT vs. $36.2 \%$ with RT; HR: 0.52, 95\% CI: 0.30-0.91), while this was not found for other molecular subgroups [9]. It is possible that the underlying aberrant p53 status in HER2-positive EC (partially) explains the promising results of combined adjuvant chemotherapy-trastuzumab in contrast to single-agent trastuzumab treatment.

Several mechanisms of trastuzumab resistance have been identified in breast cancer, one of which is increased signaling through the PI3-kinase pathway caused by gain-offunction PIK3CA mutations or inactivation of PTEN [36]. Trastuzumab resistance has been observed in primary HER2-overexpressing serous EC cell lines harboring oncogenic PIK3CA mutations [37]. In our study, pathogenic PIK3CA mutations were found in $33.9 \%$ of the cases (95\% CI: $19.8-48.0 \% ; 27.8 \%$ and $40.0 \%$ of HER2-positive EC in the PORTEC-3 trial and TCGA cohort, respectively). This is comparable to what is observed in breast cancer, in which activating PIK3CA mutations are reported in 25-38\% of HER2-positive breast cancers $[25,38,39]$. In contrast, only one HER2-positive case from PORTEC-3 and none of the ERBB2-amplified EC had a mutation in PTEN suggesting inactivation of PTEN will unlikely impact anti-HER2 therapies in HER2-positive EC. Although not much is known about the role of oncogenic PIK3CA mutations in trastuzumab resistance in HER2-positive $\mathrm{EC}$, the relatively high prevalence of the co-occurrence may be taken into consideration when designing new clinical trials on trastuzumab.

\section{Materials and Methods}

\subsection{Patient and Tissue Selection}

HER2 testing was performed on formalin-fixed paraffin-embedded (FFPE) tumor tissues molecularly profiled from 410 consenting patients included in the international PORTEC-3 clinical trial and collected by the TransPORTEC group. Detailed information on the study design and results have been described previously [2]. In brief, 660 eligible patients with high-risk EC were randomly assigned 1:1 to postoperative chemoradiotherapy 
(CTRT) versus radiotherapy (RT) alone. Pathological inclusion criteria for the PORTEC-3 trial were as follows: International Federation of Gynecology and Obstetrics (FIGO) 2009 stage IA grade 3 endometrioid EC (EEC) with documented LVSI; stage IB grade 3 EEC; stage II-III EEC; or non-endometrioid EC with stage IA (with invasion), IB, II, or III. Upfront central pathology review was performed by reference gynecopathologists to confirm eligibility. The study was approved by the Dutch Cancer and medical ethics committees at participating. Patient, tumor, and treatment characteristics were not significantly different between PORTEC-3 trial patients included in and excluded from the molecular analyses, as reported previously [9].

In addition, we used publicly available endometrial cancer data from TCGA series to further extend our cohort [40]. Clinicopathological and molecular data as well as patient outcome data from all patients with available copy number variation (CNV) data were obtained from the Genome Data Analysis Center [41].

\subsection{Immunohistochemical Staining of HER2}

Immunohistochemical (IHC) staining of HER2 was performed using Ventana BenchMark GX (Roche Diagnostics, Basel, Switzerland). Available FFPE blocks were cut into $4 \mu \mathrm{m}$ slides and stained for HER2 IHC using the anti-HER2/neu (4B5) Rabbit Monoclonal Primary Antibody and the Ventana ultraView DAB Detection Kit. Because of differences in fixation time of the tumor specimens, due to the multi-centered character of the PORTEC-3 trial, the cell conditioning step was prolonged, as compared to the standard manufacturer's protocol, to ensure optimal antibody retrieval for all samples. In addition, the incubation time of hematoxylin counterstaining was prolonged to $8 \mathrm{~min}$ for better visualization of cell nuclei. A positive control was mounted on each individual slide.

\subsection{Evaluation of HER2 IHC Staining}

HER2 IHC stained slides were reviewed by an expert gynecopathologist (T.B.)blinded to clinicopathological and molecular data-to determine the presence and intensity of membranous HER2 immunoreactivity (absent, faint, moderate, and strong) as well as the percentage of tumor cells showing membranous HER2 staining. Representative examples of faint, moderate, and strong membranous staining are shown in Figure S2. All slides with absent HER2 staining - in the presence of a positive control—as well as slides with faint membranous HER2 staining - regardless of the percentage of tumor cells and completeness of membranous staining - were classified as HER2-negative. Based on preliminary results of HER2 IHC and DISH in EC prior to this study as well as experience from diagnostic cases, we felt confident to classify these cases as HER2-negative. To confirm this approach, DISH was performed on a random selection of 20 tumors with absent or faint membranous staining. Tumors with any moderate or strong (in)complete membranous HER2 staining were considered potentially HER2-amplified and final HER2 amplification status of these cases was determined by dual in situ hybridization (DISH).

\subsection{HER2 Dual In Situ Hybridization}

DISH was performed to determine the HER2 amplification status of all cases with moderate to strong membranous HER2 IHC expression regardless of completeness of membranous staining and the percentage of tumor cells. It has been shown that HER2amplified EC frequently show incomplete membranous HER2 IHC staining [18]. Therefore, the completeness of membranous staining was not considered a criterium for subsequent DISH testing. In addition, no threshold was applied to the percentage of stained tumor cells with moderate or strong membranous staining needed to perform DISH, as such a threshold is not yet validated in EC. HER2 amplification status of all cases that failed for IHC $(n=13)$ were tested by DISH, as well as a randomly selected subset of tumors with absent $(n=10)$ or faint $(n=10)$ membranous IHC expression to confirm the lack of amplification in cases with these staining patterns. Although validated HER2 testing guidelines exist for directing conformational ISH testing in breast and gastroesophageal 
cancer, these guidelines have not been properly validated in EC [33,42]. Therefore, for the purpose of this study, we apprehended a low threshold for additional ISH testing with the intention not to miss any HER2-amplified EC. The INFORM HER2 Dual ISH (DISH) DNA Probe Cocktail assay was performed on Ventana BenchMark GX (Roche Diagnostics, Basel, Switzerland). The DISH assay was initially performed using the manufacturer's recommended protocol. However, a pre-treatment baking step was added to the protocol to prevent nuclear bubbling caused by excessive paraffin. For each slide, visible HER2 probe (black) and CEP17 (red) signals were counted in at least 20 nuclei by standard light microscopy and the HER2:CEP17 ratio was calculated. In line with the ASCO/CAP guideline recommendations for breast cancer, ERBB2 amplification was defined as the HER2:CEP17 ratio $\geq 2.0$ [33]. Since significant heterogeneity of HER2 IHC expression in EC has been reported [43], for every case, the HER2 IHC slide was consulted to identify the area(s) in the tumor in which the HER2:CEP17 ratio needed to be scored in order to prevent possible false-negative results.

\subsection{Next-Generation Sequencing}

For the purpose of previous publications, targeted next-generation sequencing (NGS) using AmpliSeq Cancer Hotspot Panel v5 was performed on PORTEC-3 tumor samples. Detailed description of DNA isolation and sequencing was described previously [9]. In cases with a failed DISH assay, the final HER2 status was determined by visual examination of copy number plots generated for every individual sample for the presence of unequivocal $E R B B 2$ amplification. All cases with low-quality NGS data were considered ineligible for the examination of $E R B B 2$ amplification.

The presence of pathogenic PIK3CA and PTEN mutations was evaluated independent of and blinded for HER2 status. A minimum coverage threshold of 100 reads and variant allele frequency of 0.1 were considered. Pathogenicity of non-synonymous PIK3CA and PTEN mutations was assessed using the public databases COSMIC [44] and ClinVar [45], as well as in silico tools SIFT [46] and PolyPhen [47]. Only mutations classified as (likely) pathogenic were included in this study.

\subsection{Statistical Analysis}

Statistical analyses were performed with SPSS (Statistical Package of Social Science) version 25 (IBM, Armonk, NY, USA). Associations between groups were analyzed using the $\chi^{2}$ statistics or Fisher's exact test for categorical variables, and the Mann-Whitney U test for continuous variables. The Spearman's Rho correlation coefficient was calculated to compare the correlations between HER2 amplification and histological and molecular characteristics. The difference between the correlations was tested by transforming the correlations to z-scores; then, we calculated the ratio of the difference to the standard error and compared this ratio to the standard normal distribution [48]. Five-year recurrence-free survival (RFS) and overall survival (OS) were estimated using the Kaplan-Meier's methodology and compared between groups using the log-rank test. To determine whether HER2 status has prognostic value independent of the established prognostic features, multivariable regression analysis using the Cox proportional hazards models was performed with the following pre-specified covariates: age, HER2 status (positive vs. negative), molecular subgroup (MMRd vs. POLEmut vs. NSMP vs. p53abn), histology (EEC grade 1-2 vs. EEC grade 3 or mixed vs. NEEC), stage (stage I-II vs. III), LVSI (absent vs. present), and adjuvant treatment received (CTRT vs. RT). Median duration of follow-up was estimated by the reversed Kaplan-Meier method [49]. A two-sided $p$-value $<0.05$ was considered statistically significant.

\section{Conclusions}

This study is the first to describe a very strong correlation between HER2 positivity and the p53abn molecular subgroup within a cohort of HREC. This finding supports molecular subgroup-directed testing of HER2 status, which is superior to histologic subtype-directed 
testing. Future clinical trials investigating trastuzumab (or other anti-HER2 therapies) should therefore screen patients with p53abn EC for HER2 positivity to determine eligibility.

Supplementary Materials: The following are available online at https:/ / www.mdpi.com/2072-6 694/13/1/44/s1, Figure S1: Kaplan-Meier survival curves for (A) recurrence-free survival (RFS) and (B) overall survival (OS) in patients with HER2-positive and HER2-negative endometrial cancer (EC) from the combined PORTEC-3 and TCGA UCEC cohorts; (C) RFS and (D) OS for patients with HER2-positive and HER2-negative p53-abnormal EC, Figure S2: Representative examples of faint, moderate and strong membranous HER2 immunoreactivity in endometrial cancer, Table S1: Multivariable analysis of HER2 status and clinicopathological features in the combined PORTEC-3 and TCGA UCEC cohorts, Table S2: Comparison of HER2 status assessed by immunohistochemistry, scored by adjusted ASCO/CAP 2007 breast cancer guideline for HER2 testing, and dual in situ hybridization.

Author Contributions: Conceptualization, L.V., N.H., V.T.S., C.L.C., T.B.; methodology, L.V., V.T.S., T.B.; formal analysis, L.V., N.H.; investigation, L.V., T.A.R., T.B.; data curation, L.V., T.A.R., T.B.; writing-original draft preparation, L.V., N.H., V.T.S., C.L.C., T.B.; writing-review and editing, L.V., N.H., A.L.-C., L.R.M., H.J.M., A.L., M.E.P., N.S., E.J.C., V.T.S., C.L.C., T.B.; visualization, L.V.; supervision, T.B.; project administration, L.V., T.B.; funding acquisition, V.T.H.B.M.S., C.L.C., T.B. All authors have read and agreed to the published version of the manuscript.

Funding: This work was supported by Dutch Cancer Society research project grants (T.B., 11629). E.J.C. is supported by the Manchester National Institute for Health Research Biomedical Research Centre (IS-BRC-1215-20007).

Institutional Review Board Statement: The study was conducted according to the guidelines of the Declaration of Helsinki, and approved by the Ethics Committee of the Dutch Cancer Society (ethic code: CKTO 2006-4) and medical ethics committees at participating centers in 2006 (ethic codes: P06.031 (CME LUMC), C7925/A8659 CCTAAC, UK), NCIC CTG EN 7 CNCIC CTG) and FEDEQYN 01/0904 (UNICANCER)).

Informed Consent Statement: Informed consent was obtained from all subjects involved in the study.

Data Availability Statement: The PORTEC-3 data presented in this study are available on request from the corresponding author. Publicly available The Cancer Genome Atlas datasets were analyzed in this study. This data can be found here: http://www.broadinstitute.org/cancer/cga.

Acknowledgments: The authors thank all members of the TransPORTEC group. We thank Nienke Solleveld (Department of Pathology, Leiden University Medical Center, Leiden, Netherlands) for her excellent help with the NGS analysis. We thank Natalja ter Haar (Department of Pathology, Leiden University Medical Center, Leiden, Netherlands) for her technical support.

Conflicts of Interest: The authors declare no conflict of interest.

\section{References}

1. Colombo, N.; Creutzberg, C.L.; Querleu, D.; Barahona, M.; Sessa, C.; Committee, E.G. Appendix 5: Endometrial cancer: eUpdate published online 8 June 2017 (www.esmo.org/Guidelines/Gynaecological-Cancers). Ann. Oncol. 2017, 28, iv153-iv156. [CrossRef] [PubMed]

2. De Boer, S.; Powell, M.E.; Mileshkin, L.; Katsaros, D.; Bessette, P.; Haie-Meder, C.; Ottevanger, P.B.; Ledermann, J.A.; Khaw, P.; Colombo, A.; et al. Adjuvant chemoradiotherapy versus radiotherapy alone for women with high-risk endometrial cancer (PORTEC-3): Final results of an international, open-label, multicentre, randomised, phase 3 trial. Lancet Oncol. 2018, 19, 295-309. [CrossRef]

3. Gilks, C.B.; Oliva, E.; Soslow, R.A. Poor interobserver reproducibility in the diagnosis of high-grade endometrial carcinoma. Am. J. Surg. Pathol. 2013, 37, 874-881. [CrossRef] [PubMed]

4. Bendifallah, S.; Canlorbe, G.; Collinet, P.; Arsene, E.; Huguet, F.; Coutant, C.; Hudry, D.; Graesslin, O.; Raimond, E.; Touboul, C.; et al. Just how accurate are the major risk stratification systems for early-stage endometrial cancer? Br. J. Cancer 2015, 112, 793-801. [CrossRef] [PubMed]

5. Talhouk, A.; McConechy, M.K.; Leung, S.; Li-Chang, H.H.; Kwon, J.S.; Melnyk, N.; Yang, W.; Senz, J.; Boyd, N.F.; Karnezis, A.N.; et al. A clinically applicable molecular-based classification for endometrial cancers. Br. J. Cancer 2015, 113, 299-310. [CrossRef] [PubMed]

6. Talhouk, A.; McConechy, M.K.; Leung, S.; Yang, W.; Lum, A.; Senz, J.; Boyd, N.; Pike, J.; Anglesio, M.; Kwon, J.; et al. Confirmation of ProMisE: A simple, genomics-based clinical classifier for endometrial cancer. Cancer 2017, 123, 802-813. [CrossRef] [PubMed] 
7. Kommoss, S.; McConechy, M.; Leung, S.; Bunz, A.; Magrill, J.; Britton, H.; Grevenkamp, F.; Karnezis, A.; Yang, W.; Lum, A.; et al. Final validation of the ProMisE molecular classifier for endometrial carcinoma in a large population-based case series. Ann. Oncol. 2018, 29, 1180-1188. [CrossRef]

8. Stelloo, E.; Nout, R.A.; Osse, E.M.; Juergenliemk-Schulz, I.J.; Jobsen, J.J.; Lutgens, L.C.; Van Der Steen-Banasik, E.M.; Nijman, H.W.; Putter, H.; Bosse, T.; et al. Improved risk assessment by integrating molecular and clinicopathological factors in early-stage endometrial cancer-Combined analysis of the PORTEC cohorts. Clin. Cancer Res. 2016, 22, 4215-4224. [CrossRef]

9. León-Castillo, A.; De Boer, S.M.; Powell, M.E.; Mileshkin, L.R.; Mackay, H.J.; Leary, A.; Nijman, H.W.; Singh, N.; Pollock, P.M.; Bessette, P.; et al. Molecular classification of the PORTEC-3 trial for high-risk endometrial cancer: Impact on prognosis and benefit from adjuvant therapy. J. Clin. Oncol. 2020. [CrossRef]

10. Rüschoff, J.; Dietel, M.; Baretton, G.; Arbogast, S.; Walch, A.; Monges, G.; Chenard, M.-P.; Penault-Llorca, F.; Nagelmeier, I.; Schlake, W.; et al. HER2 diagnostics in gastric cancer-guideline validation and development of standardized immunohistochemical testing. Virchows Arch. 2010, 457, 299-307. [CrossRef]

11. Hofmann, M.; Stoss, O.; Shi, D.; Büttner, R.; Van De Vijver, M.; Kim, W.; Ochiai, A.; Rüschoff, J.; Henkel, T. Assessment of a HER2 scoring system for gastric cancer: Results from a validation study. Histopathology 2008, 52, 797-805. [CrossRef]

12. Grávalos, C.; Jimeno, A. HER2 in gastric cancer: A new prognostic factor and a novel therapeutic target. Ann. Oncol. 2008, 19, 1523-1529. [CrossRef] [PubMed]

13. Ross, J.S.; Fletcher, J.A.; Linette, G.P.; Stec, J.; Clark, E.; Ayers, M.; Symmans, W.F.; Pusztai, L.; Bloom, K.J. The HER-2/neu gene and protein in breast cancer 2003: Biomarker and target of therapy. Oncologist 2003, 8, 307-325. [CrossRef] [PubMed]

14. Slamon, D.J.; Leyland-Jones, B.; Shak, S.; Fuchs, H.; Paton, V.; Bajamonde, A.; Fleming, T.; Eiermann, W.; Wolter, J.; Pegram, M.; et al. Use of chemotherapy plus a monoclonal antibody against HER2 for metastatic breast cancer that overexpresses HER2. N. Engl. J. Med. 2001, 344, 783-792. [CrossRef] [PubMed]

15. Piccart, M.; Procter, M.; Leyland-Jones, B.; Goldhirsch, A.; Untch, M.; Smith, I.; Gianni, L.; Baselga, J.; Bell, R.H.; Jackisch, C.; et al. Trastuzumab after adjuvant chemotherapy in HER2-positive breast cancer. N. Engl. J. Med. 2005, 353, 1659-1672. [CrossRef] [PubMed]

16. Smith, I.; Procter, M.; Gelber, R.D.; Guillaume, S.; Feyereislova, A.; Dowsett, M.; Goldhirsch, A.; Untch, M.; Mariani, G.; Baselga, J.; et al. 2-year follow-up of trastuzumab after adjuvant chemotherapy in HER2-positive breast cancer: A randomised controlled trial. Lancet 2007, 369, 29-36. [CrossRef]

17. Bang, Y.J.; Van Cutsem, E.; Feyereislova, A.; Chung, H.C.; Shen, L.; Sawaki, A.; Lordick, F.; Ohtsu, A.; Omuro, Y.; Satoh, T.; et al. Trastuzumab in combination with chemotherapy versus chemotherapy alone for treatment of HER2-positive advanced gastric or gastro-oesophageal junction cancer (ToGA): A phase 3, open-label, randomised controlled trial. Lancet 2010, 376, 687-697. [CrossRef]

18. Buza, N.; English, D.P.; Santin, A.D.; Hui, P. Toward standard HER2 testing of endometrial serous carcinoma: 4-year experience at a large academic center and recommendations for clinical practice. Mod. Pathol. 2013, 26, 1605-1612. [CrossRef]

19. Halle, M.K.; Tangen, I.L.; Berg, H.F.; Hoivik, E.A.; Mauland, K.K.; Kusonmano, K.; Berg, A.; Hurtado, A.; Kalland, K.H.; Øyan, A.M.; et al. HER2 expression patterns in paired primary and metastatic endometrial cancer lesions. Br. J. Cancer 2018, 118, 378-387. [CrossRef]

20. Morrison, C.; Zanagnolo, V.; Ramirez, N.; Cohn, D.E.; Kelbick, N.; Copeland, L.; Maxwell, L.G.; Fowler, J.M. HER-2 is an independent prognostic factor in endometrial cancer: Association with outcome in a large cohort of surgically staged patients. J. Clin. Oncol. 2006, 24, 2376-2385. [CrossRef]

21. Konecny, G.E.; Santos, L.; Winterhoff, B.; Hatmal, M.; Keeney, G.L.; Mariani, A.; Jones, M.; Neuper, C.; Thomas, B.; Muderspach, L.; et al. HER2 gene amplification and EGFR expression in a large cohort of surgically staged patients with nonendometrioid (type II) endometrial cancer. Br. J. Cancer 2009, 100, 89-95. [CrossRef]

22. Fader, A.N.; Roque, D.M.; Siegel, E.; Buza, N.; Hui, P.; Abdelghany, O.; Chambers, S.K.; Secord, A.A.; Havrilesky, L.J.; O'Malley, D.M.; et al. Randomized phase II trial of carboplatin-paclitaxel versus carboplatin-paclitaxel-trastuzumab in uterine serous carcinomas that overexpress human epidermal growth factor receptor 2/neu. J. Clin. Oncol. 2018, 36, $2044-2051$. [CrossRef] [PubMed]

23. Fader, A.N.; Roque, D.M.; Siegel, E.R.; Buza, N.; Hui, P.; Abdelghany, O.; Chambers, S.; Secord, A.A.; Havrilesky, L.; O'Malley, D.M.; et al. Randomized phase II trial of carboplatin-paclitaxel compared with carboplatin-paclitaxel-trastuzumab in advanced (Stage III-IV) or recurrent uterine serous carcinomas that overexpress her2/neu (NCT01367002): Updated overall survival analysis. Clin. Cancer Res. 2020, 26, 3928-3935. [CrossRef]

24. Loibl, S.; Von Minckwitz, G.; Schneeweiss, A.; Paepke, S.; Lehmann, A.; Rezai, M.; Zahm, D.M.; Sinn, P.; Khandan, F.; Eidtmann, H.; et al. PIK3CA mutations are associated with lower rates of pathologic complete response to anti-human epidermal growth factor receptor 2 (HER2) therapy in primary HER2-overexpressing breast cancer. J. Clin. Oncol. 2014, 32, 3212-3220. [CrossRef]

25. Berns, K.; Horlings, H.M.; Hennessy, B.T.; Madiredjo, M.; Hijmans, E.M.; Beelen, K.; Linn, S.C.; Gonzalez-Angulo, A.M.; Stemke-Hale, K.; Hauptmann, M.; et al. A functional genetic approach identifies the PI3K pathway as a major determinant of trastuzumab resistance in breast cancer. Cancer Cell 2007, 12, 395-402. [CrossRef] [PubMed] 
26. Nagata, Y.; Lan, K.-H.; Zhou, X.; Tan, M.; Esteva, F.J.; Sahin, A.A.; Klos, K.S.; Li, P.; Monia, B.P.; Nguyen, N.T.; et al. PTEN activation contributes to tumor inhibition by trastuzumab, and loss of PTEN predicts trastuzumab resistance in patients. Cancer Cell 2004, 6, 117-127. [CrossRef] [PubMed]

27. WHO Classification of Tumours Editorial Board (Ed.) Female Genital Tumours, 5th ed.; WHO Press: Lyon, France, 2020.

28. Román-Rosales, A.A.; García-Villa, E.; Herrera, L.A.; Gariglio, P.; Díaz-Chávez, J. Mutant p53 gain of function induces HER2 over-expression in cancer cells. BMC Cancer 2018, 18, 709. [CrossRef] [PubMed]

29. Fedorova, O.; Daks, A.; Shuvalov, O.; Kizenko, A.; Petukhov, A.; Gnennaya, Y.; Barlev, N.A. Attenuation of p53 mutant as an approach for treatment Her2-positive cancer. Cell Death Discov. 2020, 6, 100. [CrossRef] [PubMed]

30. Li, D.; Yallowitz, A.; Ozog, L.; Marchenko, N.D. A gain-of-function mutant p53-HSF1 feed forward circuit governs adaptation of cancer cells to proteotoxic stress. Cell Death Dis. 2014, 5, e1194. [CrossRef]

31. Bull, S.B.; Ozcelik, H.; Pinnaduwage, D.; Blackstein, M.E.; Sutherland, D.A.; Pritchard, K.I.; Tzontcheva, A.T.; Sidlofsky, S.; Hanna, W.M.; Qizilbash, A.H.; et al. The Combination of p53 mutation and neu/erbB-2 amplification is associated with poor survival in node-negative breast cancer. J. Clin. Oncol. 2004, 22, 86-96. [CrossRef]

32. Buza, N.; Roque, D.M.; Santin, A.D. HER2/neu in endometrial cancer: A promising therapeutic target with diagnostic challenges. Arch. Pathol. Lab. Med. 2014, 138, 343-350. [CrossRef] [PubMed]

33. Wolff, A.C.; Hammond, M.E.H.; Allison, K.H.; Harvey, B.E.; Mangu, P.B.; Bartlett, J.M.S.; Bilous, M.; Ellis, I.O.; Fitzgibbons, P.; Hanna, W.; et al. Human epidermal growth factor receptor 2 testing in breast cancer: American society of clinical oncology/college of american pathologists clinical practice guideline focused update. J. Clin. Oncol. 2018, 36, 2105-2122. [CrossRef] [PubMed]

34. Fleming, G.F.; Sill, M.W.; Darcy, K.M.; McMeekin, D.S.; Thigpen, J.T.; Adler, L.M.; Berek, J.S.; Chapman, J.A.; DiSilvestro, P.A.; Horowitz, I.R.; et al. Phase II trial of trastuzumab in women with advanced or recurrent, HER2-positive endometrial carcinoma: A Gynecologic Oncology Group study. Gynecol. Oncol. 2010, 116, 15-20. [CrossRef] [PubMed]

35. Koskas, M.; Depreeuw, J.; Moens, S.; Annibali, D.; Cuppens, T.; Moerman, P.; Lambrechts, D.; Amant, F. Genomic characterisation and response to trastuzumab and paclitaxel in advanced or recurrent HER2-positive endometrial carcinoma. Anticancer. Res. 2016, 36, 5381-5384. [CrossRef]

36. Diver, E.J.; Foster, R.; Rueda, B.R.; Growdon, W.B. The therapeutic challenge of targeting HER2 in endometrial cancer. Oncologist 2015, 20, 1058-1068. [CrossRef] [PubMed]

37. Black, J.D.; Lopez, S.; Cocco, E.; Bellone, S.; Altwerger, G.; Schwab, C.L.; English, D.P.; Bonazzoli, E.; Predolini, F.; Ferrari, F.; et al. PIK3CA oncogenic mutations represent a major mechanism of resistance to trastuzumab in HER2/neu overexpressing uterine serous carcinomas. Br. J. Cancer 2015, 113, 1641. [CrossRef] [PubMed]

38. Li, S.Y.; Rong, M.; Grieu, F.; Iacopetta, B. PIK3CA mutations in breast cancer are associated with poor outcome. Breast Cancer Res. Treat. 2006, 96, 91-95. [CrossRef]

39. Saal, L.H.; Holm, K.; Maurer, M.; Memeo, L.; Su, T.; Wang, X.; Yu, J.S.; Malmström, P.-O.; Mansukhani, M.; Enoksson, J.; et al. PIK3CA mutations correlate with hormone receptors, node metastasis, and ERBB2, and are mutually exclusive with PTEN loss in human breast carcinoma. Cancer Res. 2005, 65, 2554-2559. [CrossRef]

40. Cancer Genome Atlas Research Network; Kandoth, C.; Schultz, N.; Cherniack, A.D.; Akbani, R.; Liu, Y.; Shen, H.; Robertson, A.G.; Pashtan, I.; Shen, R.; et al. Integrated genomic characterization of endometrial carcinoma. Nature 2013, 497, 67-73. [CrossRef]

41. Genome Data Analysis Center. Available online: http://www.broadinstitute.org/cancer/cga (accessed on 3 July 2020).

42. Bartley, A.N.; Washington, M.K.; Ventura, C.B.; Ismaila, N.; Colasacco, C.; Benson, A.B.; Carrato, A.; Gulley, M.L.; Jain, D.; Kakar, S.; et al. HER2 Testing and Clinical Decision Making in Gastroesophageal Adenocarcinoma: Guideline From the College of American Pathologists, American Society for Clinical Pathology, and American Society of Clinical Oncology. Am. J. Clin. Pathol. 2016, 146, 647-669. [CrossRef]

43. Buza, N.; Hui, P. Marked heterogeneity ofHER2/NEUgene amplification in endometrial serous carcinoma. Genes Chromosom. Cancer 2013, 52, 1178-1186. [CrossRef] [PubMed]

44. Forbes, S.A.; Beare, D.; Gunasekaran, P.; Leung, K.; Bindal, N.; Boutselakis, H.; Ding, M.; Bamford, S.; Cole, C.; Ward, S.; et al. COSMIC: Exploring the world's knowledge of somatic mutations in human cancer. Nucleic Acids Res. 2015, 43, D805-D811. [CrossRef] [PubMed]

45. Landrum, M.J.; Lee, J.M.; Riley, G.R.; Jang, W.; Rubinstein, W.S.; Church, D.M.; Maglott, D.R. ClinVar: Public archive of relationships among sequence variation and human phenotype. Nucleic Acids Res. 2014, 42, D980-D985. [CrossRef] [PubMed]

46. Kumar, P.; Henikoff, S.; Ng, P.C. Predicting the effects of coding non-synonymous variants on protein function using the SIFT algorithm. Nat. Protoc. 2009, 4, 1073-1081. [CrossRef]

47. Adzhubei, I.A.; Schmidt, S.; Peshkin, L.; Ramensky, V.E.; Gerasimova, A.; Bork, P.; Kondrashov, A.S.; Sunyaev, S.R. A method and server for predicting damaging missense mutations. Nat Methods 2010, 7, 248-249. [CrossRef]

48. Snedecor, G.W.; Cochran, W.G. Statistical Methods; The Iowa State University Press: Ames, IA, USA, 1980.

49. Schemper, M.; Smith, T.L. A note on quantifying follow-up in studies of failure time. Control. Clin. Trials 1996, 17, 343-346. [CrossRef] 